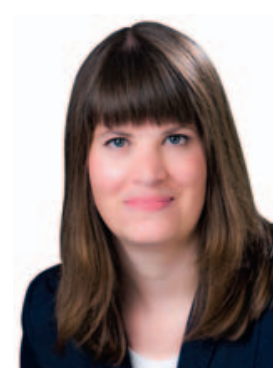

Stephanie Joachim

Koordination «OphthalmoCampus»

\section{Ophthalmologie - interaktionell, international, interdisziplinär}

Es ist äußerst erfreulich, dass der «OphthalmoCampus» im Karger Kompass Ophthalmologie nun bereits in das zweite Erscheinungsjahr geht. Wir freuen uns, für das kommende Jahr wieder spannenden Themen und Beiträge gefunden zu haben, die für Sie hoffentlich von großem Interesse sind.

In der ersten Ausgabe für dieses Jahr erhalten wir von Dr. Frings von der UniversitätsAugenklinik in Düsseldorf eine sehr gute Übersicht zum Thema «Rote Augen bei Kontaktlinsenträgern» und dazu, was bei diesen Notfällen zu tun ist. Dr. Maximilian Wintergerst aus Bonn hat, in Zusammenarbeit mit dem Sankara Eye Center in Bangalore, Indien, eine Smartphone-Funduskopie konzipiert. Er berichtet, wie sie diese einfache und kostengünstige Funduskopie alltagstauglich machen wollen.

Anschließend berichtet Prof. Axel W. Bauer, der einen Ratgeber für Mediziner geschrieben hat, die promovieren möchten, über seine Erfahrungen und Eindrücke zur medizinischen Promotion.

Auf dem Herbstreffen des Bündnis Junger Ärzte (BJÄ) in München wurde über Zeitprobleme im ärztlichen Alltag diskutiert. Der Beitrag von Fr. Bauer fasst die Gesprächsrunde der Jungen Ärzte zusammen.

Abschließend wird über die Notwendigkeit von Fachverlagen für junge Wissenschaftler von Vertretern verschiedener Fachdisziplinen diskutiert.

Bei der Lektüre des aktuellen «OphthalmoCampus» wünsche ich viel Vergnügen und alles Gute für das neue Jahr!

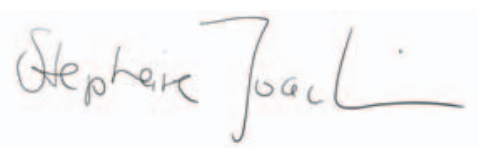

\section{KARGER}

Fax +497614520714

information@karger.com www.karger.com (c) 2018 S. Karger GmbH, Freiburg
PD Dr. Stephanie Joachim

Head of Experimental Eye Research

In der Schornau 23-25, 44892 Bochum, Deutschland

stephanie.joachim@rub.de 


\section{Ophthalmologie kompakt}

\section{Vorsicht bei rotem Auge bei Kontaktlinsenträgern}

Das rote Auge ist Leitsymptom multipler Erkrankungen und daher eine tägliche diagnostische Herausforderung in der (ophthalmologischen) Sprechstunde [1-3]. Auch innerhalb der hausärztlichen und Notfallversorgung macht das rote Augen circa 3\% der täglichen Konsultationen aus [1].

Die häufigste Ursache für rote Augen ist die Konjunktivitis. Infektiöse Konjunktividen sind in der Regel bakteriell oder viral. Im deutschsprachigen Raum sind die häufigsten Erreger einer bakteriellen Konjunktivitis Staphylo-, Strepto- oder Pneumokokken [4-6]. Neben einer starken Rötung der Augen sind Schmerzen und ein mukopurolentes Sekret mit gelblicher Krustenbildung typische Zeichen.

Bei Kontaktlinsenträgern stehen andere Erreger im Vordergrund, hier findet man häufiger Infektionen mit Akanthamöben oder Pseudomonas aeruginosa [7, 8]. Die perakute Infektion mit Pseudomonaden kann dra-

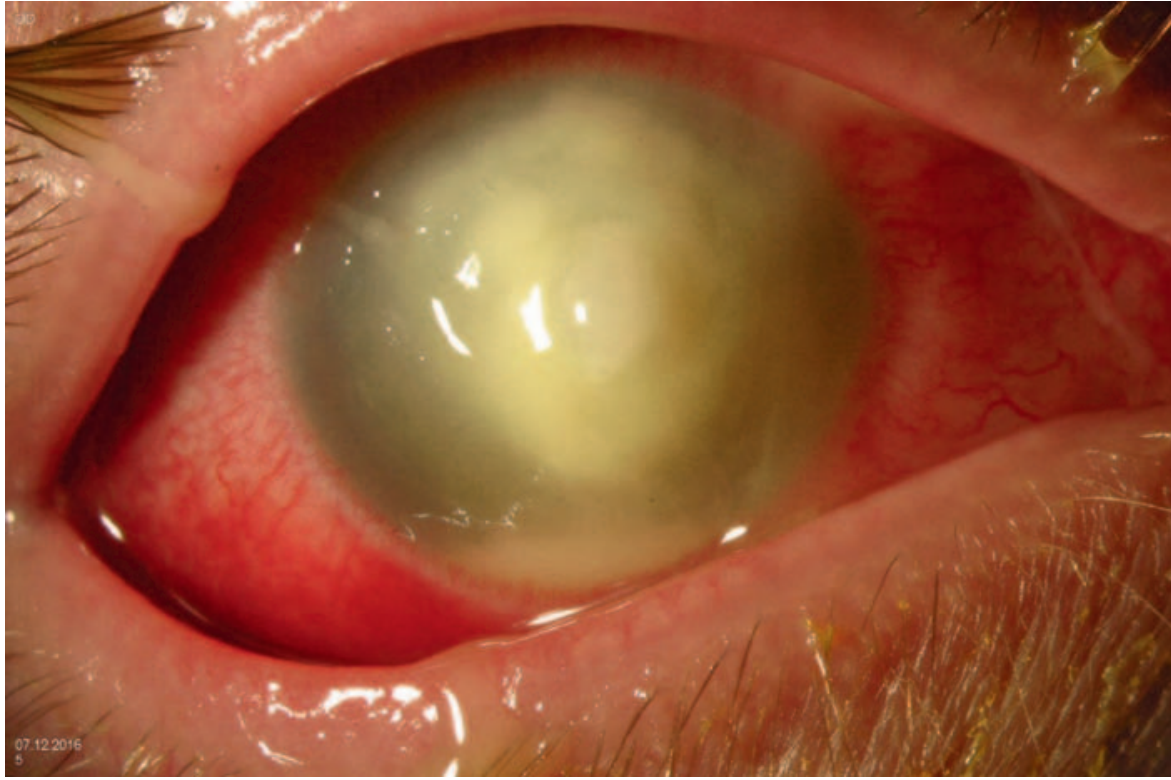

Abb.1. Rechtes Auge eines Patienten mit weit fortgeschrittener mykotischer Keratitis. Man erkennt die zentral durch den Pilz infiltrierte Hornhaut mit sekundärer Eiteransammlung am Boden der dahinterliegenden Vorderkammer. Dieser Befund entwickelte sich über mehrere Wochen aufgrund einer initial fälschlicherweise als bakterielle Keratitis therapierten Pilzinfektion. matisch verlaufen; innerhalb von wenigen Stunden kann es zu einer Ausbildung eines tiefen Hornhautulkus kommen. Der Erregernachweis gelingt hier häufig nicht primär aus dem Ulkusabstrich oder dem Hornhaut-Scraping, zielführender ist der zusätzliche Erregernachweis aus den Aufbewahrungsflüssigkeiten/-behältnissen der Kontaktlinsen. Therapeutische Hürden bestehen aufgrund häufiger Resistenzen gegen gängige Antibiotika. Im Falle eines roten Auges bei Kontaktlinsenträgern ist eine zeitnahe Diagnosesicherung mittels Erregernachweis daher besonders wichtig. Hierzu ist die Bereitstellung des ungesäuberten Kontaktlinsenbehälters samt Flüssigkeit und Linse sowie ein direkter Abstrich vom Entzündungsherd durch den Ophthalmologen im Rahmen einer sofortigen augenärztlichen Vorstellung unumgänglich.

Nicht adäquat therapierte mikrobielle Keratokunjunktivitiden können in Einzelfällen sogar zur Ausbildung perforierender Hornhautulcera mit nachhaltig schwerwiegenden Schäden oder gar zum Verlust des Auges führen [7]. Eine mögliche Therapieoption ist dann nur noch eine Keratoplastik à chaud [8].

Die Pilz-Keratitis (mykotische Keratitis) ist ein seltenes, aber sehr ernst zu nehmendes ophthalmologisches Krankheitsbild mit ebenfalls potenziell visus-bedrohendem Verlauf (Abb. 1).

Infektionen durch Pilze zeigen häufig einen protrahierten und destruktiven Verlauf, was nicht zuletzt an der schwierigen Diagnosesicherung und korrekten Therapieeinleitung liegt. Aufgrund der vielfältigen Erscheinungsbilder der kornealen Pilzerkrankungen ist selbst für den geschulten Kliniker die Diagnose nach wie vor ein großes Problem [9]. Mit Hilfe der kornealen konfokalen Mikroskopie kann in einigen Fällen die Darstellung der Pilzhyphen gelingen und so die Diagnose frühzeitig bestätigt werden. Die frühzeitige adäquate Be- handlung ist bei Pilzkeratitiden ein besonders wichtiger prognostischer Faktor [10]. Möglicherweise bedingt durch die vermehrte Verwendung weicher Kontaktlinsen ist in den letzten Jahren eine Häufigkeitszunahme von Pilzinfektionen festzustellen. In den letzten Jahren wurden auch neue humanpathogene Pilzarten dokumentiert. Wir behandelten an der Universitätsaugenklinik in Düsseldorf kürzlich den nach der Erstbeschreibung im Jahr 2016 weltweit zweiten Fall einer mikrobiellen Keratitis durch Tintelnotia destructans, einem Erreger, der als Ursache der Onychomykose (Nagelpilz) bekannt ist [11]. Um eine besonders effiziente Diagnostik bei unklaren Keratitiden oder bei klinischem Verdacht auf Pilzinfektionen sicherzustellen, wurde daher das deutsche Register für mykotische Keratitiden auf gemeinsame Initiative des Nationale Referenzzentrum für invasive Pilzinfektionen (NRZMyk) und der Universitätsaugenklinik Düsseldorf mit Unterstützung der Sektion Kornea der Deutschen Ophthalmologischen Gesellschaft (DOG) etabliert [12].

\section{Fazit für die Praxis}

- Rote Augen bei Kontaktlinsenträgern sind immer ein ophthalmologischer Notfall und müssen daher binnen 12 Stunden augenärztlich vorgestellt werden.

- Mitzubringen sind die Kontaktlinsen und der «native» Kontaktlinsenbehälter samt Flüssigkeit.

- Eine mykotische Keratitis ist eine potentiell organbedrohende Erkrankung, deren Häufigkeitszunahme auch durch die Anwendung weicher Kontaktlinsen begünstigt wird.

Dr. Dr. Andreas Frings, MHBA (Univ.-Augenklinik Düsseldorf) \& PD Dr. Marc Schargus, MHBA, FEBO (Univ.-Augenklinik Düsseldorf) 
1 Pflipsen M, Massaquoi M, Wolf S: Evaluation of the painful eye. AmFam Physician 2016;93:991-998.

2 Teo MA: Improving acute eye consultations in general practice: a practical approach. BMJ Qual Improv Rep 2014:3;DOI:10.1136/bmjquality.u206617.w2852.

3 Cronau H, Kankanala RR, Mauger T: Diagnosis and management of red eye in primary care. Am Fam Physician 2010;81:137-44.

4 Azari AA, Barney NP: Conjunctivitis: a systematic review of diagnosis and treatment. JAMA 2013;310:1721-1729.

5 Alfonso SA, Fawley JD, Alexa Lu X: Conjunctivitis. Prim Care 2015;42:325-345.

6 Messmer EM: Keratitis - infectious or autoimmune? Klin Monbl Augenheilkd 2016; 233:808-812.

7 Fernandes M, Vira D, Medikonda R, Kumar N: Extensively and pandrug resistant pseudomonas aeruginosa keratitis: clinical features, risk factors, and outcome. Graefes Arch Clin Exp Ophthalmol 2016;254:315-322.

8 Vazirani J, Wurity S, Ali MH: Multidrug-resistant pseudomonas aeruginosa keratitis: risk factors, clinical characteristics, and outcomes. Ophthalmology 2015;122: $2110-2114$.
9 Jurkunas U, Behlau I, Colby K: Fungal keratitis: changing pathogens and risk factors. Cornea. 2009;28:638-643.

10 Ansari Z, Miller D, Galor A: Current thoughts in fungal keratitis: diagnosis and treatment. Curr Fungal Infect Rep 2013;7:209-218.

11 Ahmed SA, Hofmuller W, Seibold M, et al.: Tintelnotia, a new genus in Phaeosphaeriaceae harbouring agents of cornea and nail infections in humans. Mycoses 2017; 60:244-253.

12 NRZMyk, Universitätsaugenklinik Düsseldorf: Nationales Register für mykotische Keratitiden. www.pilzkeratitis.de (Zugriff: 18.12.2017).

Kontaktadresse: Dr. Andreas Frings, Universitätsklinik für Augenheilkunde der Heinrich-Heine-Universität Düsseldorf, Moorenstraße 5, 40225 Düsseldorf, Deutschland, andi.frings@gmail.com.

\section{Medizin international}

\section{Smartphone-Ophthalmoskopie in Indien - Interview mit Dr. Wintergerst}

Ungefähr jeder zehnte Mensch in Südindien leidet an Diabetes, davon hat etwajeder dritteschon eine diabetische Retinopathieentwickelt. Um die Folgen der diabetischen Retinopathie vorzubeugen, die unbehandelt zu Sehbehinderung und Blindheit führt, benötigt es eine frühzeitige Erkennung. In den medizinisch unterversorgten Gegenden außerhalb der Städte oder in Slums ist dies aber nicht ohne Weiteres möglich. Um auch diesen Menschen helfen zu können, untersuchten das Universitätsklinikum Bonn und das Sankara Eye Center in Bangalore, Indien ein Screening-Verfahren, das leicht zugänglich und kostengünstig war: Mithilfe eines Aufsatzes konnte die Kamera eines Smartphones in ein Ophthalmoskop umgewandelt werden. Geschultes Personal kann so Fotos von Patientenaugen machen und zur Auswertung an einen Augenarzt weitersenden. Dr. Maximilian Wintergerst, der dieses Screening-Verfahren untersucht, berichtet für uns im OphthalmoCampus über dieses spannende Projekt.

Woher kam die Idee für das kostengünstige Augen-Screening von Menschen mit Diabetes in Indien?

Smartphones sind in der heutigen Welt allgegenwärtig, sogar in Entwicklungs- und Schwellenländern. Viele verfügen über eine vergleichsweise gute Kamera, wären also prinzipiell für medizinische Bildgebung geeignet, allerdings ist der Strahlengang der Handykamera nicht dafür ausgelegt durch eine sehr kleine Öffnung wie die menschliche Pupille hindurch Bilder zu machen. Hier kommen verschiedene Adapter ins Spiel, die seit kurzer Zeit von unterschiedlichen Anbietern entwickelt und angeboten werden. Durch sie wird der Strahlengang des Smartphones so modifiziert, dass die Handykamera zur Funduskopie verwendet werden kann. Ziel unserer Studie war der Vergleich verschiedener technischer Ansätze zur Smart-
phone-Funduskopie hinsichtlich Bildqualität und Eignung zum Screening für die diabetische Retinopathie.

\section{Wie kam die Zusammenarbeit mit dem Sankara Eye Center in Bangalore, Indien zustande?}

Die Kollegen des Sankara Eye Hospitals in Bangalore hatten sich selbst bereits mit der Möglichkeit der Smartphone-Funduskopie beschäftigt und einen sehr einfachen und kostengünstigen Ansatz

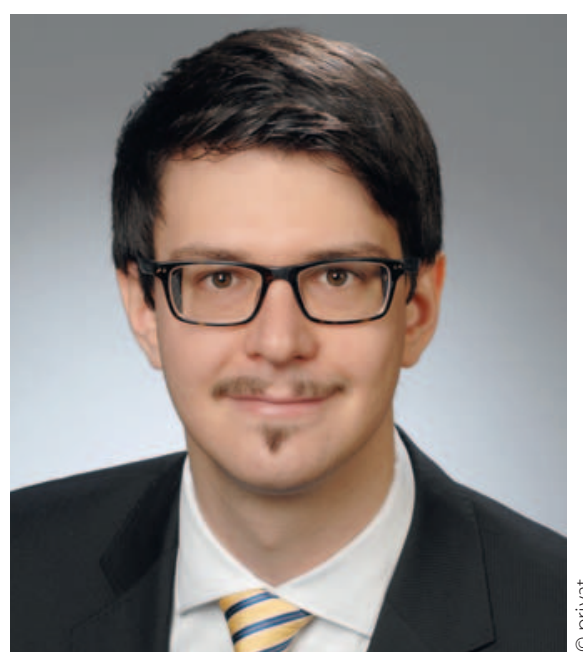

«Ich fürchtete, das komplette Geräte-Set würde am Flughafen beschlagnahmt werden.» zur Smartphone-Funduskopie entwickelt. Während kommerzielle Adapter einige hundert Euro kosten belaufen sich die Kosten für die selbstentwickelte Lösung auf etwa 50 indische Rupien, also weniger als einen Euro. Diese Vorarbeit des Sankara Eye Hospitals Bangalore war eine sehr gute Grundlage für die Kooperation, in deren Rahmen wir auch die vor Ort entwickelte sehr einfache technische Lösung mit den anderen Adaptern verglichen.

Wie wird so ein Projekt praktisch umgesetzt? Was muss man bedenken?

Die Organisation solch eines Projektes beginnt bei der Einwerbung von Fördermitteln - so wird unser Projekt durch die Forschungsförderung Tropenophthalmologie der Deutschen Ophthalmologischen Ge-

sellschaft unterstützt -, geht über Formalien wie die Bewerbung um ein Visum und beinhaltet natürlich auch Aspekte wie die Planung der Reise und Unterkunft vor Ort. Ganz entscheidend war bereits frühzeitig schon aus Deutschland den Ablauf der Studie in Bangalore zu organisieren. Gerade die organisatorischen Aspekte vor Ort sind nicht zu unterschätzen, auch weil in einem Land wie Indien einiges länger benötigt oder komplizierter ist als in Mitteleuropa. 


\section{Wie funktioniert die Smartphone-Funduskopie?}

Wir testeten drei Smartphone-Adapter zur direkten und einen zur indirekten Funduskopie. Am Beispiel einer der direkten Smartphone-Funduskopie Lösungen erklärt: Der auf das Smartphone montierte Adapter schwächt das Blitzlicht ab und lenkt es durch Spiegel auf die optische Achse der Kamera um. Mit diesem Smartphone nähert sich der Untersucher langsam an das Auge des Patienten an bis er ein scharfes Bild der Papille mit der umgebenden Retina erhält. Durch Kipp- und leichte Schwenkbewegungen der Kamera lassen sich verschiedene Bereiche des Fundus darstellen und auf die Veränderungen der diabetischen Retinopathie wie Hämorrhagien, Exsudate und Neovaskularisationen hin untersuchen.

\section{Was war die größte Herausforderung während das Projekt lief?}

Da wir für die Ausbildung der uns unterstützenden Optometristen in der Smartphone-Funduskopie und die 13 Screening Camps, in denen wir 400 Augen untersuchen mussten, nur vier Wochen in Indien zur Verfügung hatten, galt es die Zeit vor Ort möglichst effizient zu managen. Eine weitere Herausforderung war mit den speziell präparierten Smartphones mit Adaptern wieder aus Indien ausreisen zu können. Am Flughafen Bangalore fürchtete ich für kur- ze Zeit, das komplette Geräte-Set würde beschlagnahmt werden, denn die Sicherheitsbeamten waren äußerst skeptisch, was ich mit den vielen Smartphones mit seltsamen Aufsätzen vorhätte. Nachdem ich kurzerhand an einem der Sicherheitsbeamten unter den kritischen Augen seiner Kollegen vorführte, wofür die SmartphoneAdapter gut sind, konnte ich sie dann doch überzeugen.

Welchen Moment werden Sie besonders in Erinnerung behalten? Hier ist sicherlich unser Screening Camp in Bagepalli, einer kleinen Stadt etwa drei Autostunden von Bangalore entfernt zu nennen. Wir bauten unser Screening in einer lokalen Grundschule auf, wussten zu diesem Zeitpunkt allerdings noch nicht, dass die Schule von einer Horde wilder Affen okkupiert wurde. Es waren gerade Ferien, entsprechend hatten die Affen freie Hand. Bei der Inspektion des Gebäudes wurden wir auf dem Dach dann von den Makaken überrascht, die uns mit wildem Geschrei und gefletschten Zähne empfingen.

\section{Dr. Wintergerst, vielen Dank für das Interview!}

Kontaktadresse: Dr. Maximilian W. M. Wintergerst, Universitäts-Augenklinik Bonn, Ernst-Abbe-Straße 2, 53127 Bonn, Deutschland, maximilian.wintergerst@ukbonn.de.

\section{Interview mit Prof. Axel W. Bauer}

\section{Über die medizinische Promotion}

Diemeisten Medizinstudierenden wollen promovieren, doch wie geht das? Wie findet man das passende Thema, den richtigen Betreuer und wann beginnt man am besten mit der Planung? Prof. Axel W. Bauer hat an einem Ratgeber für Mediziner mitgeschrieben, die promovieren möchten. Im Interview erklärt er, wie man die eigene Promotion am besten plant und wann man das Promotionsprojekt besser als erledigt betrachten sollte.

Herr Prof. Bauer, mit welcher Problematik haben angehende Mediziner bei ihrer Promotion besonders zu kämpfen?

Jedes Kapitel unseres Ratgebers beschreibt einen wichtigen Punkt, aber ganz wesentlich erscheint mir die Frage des Zeitpunkts und der Themensuche. Denn wenn man das falsche Thema erwischt hat oder den falschen Zeitpunkt, dann wird die Sache nicht glücken.

Wann ist denn der richtige Zeitpunkt, um mit der Doktorarbeit zu beginnen?

Man sagt einerseits: nicht zu früh. Also möglichst nicht schon im Grundstudium. Ausgeschlossen ist das zwar nicht, aber der günstigere Zeitpunkt ist sicher eher in den ersten Semestern des Hauptstudiums, also im 3. oder 4. Studienjahr, wobei dann wiederum das Problem hinzukommt, dass man möglichst nach dem 5. Studienjahr fertig sein möchte. Die Zeit kann dann knapp werden, und dann zieht sich die Bearbeitung der Dissertation über das Staatsexamen hinaus.

Eine Doktorarbeit während der Weiterbildung zu verfassen halten Sie also für schwierig?

Das ist ohne Zweifel sehr schwierig. Immer wieder melden sich auch bei mir Kandidaten in diesem Berufsstadium, aber sie unter- schätzen, wie viel Arbeit eine Dissertation macht. Man muss enorm viel Mühe investieren, um Bibliotheken aufzusuchen oder auch mal ein Archiv, oder um Experimente zu machen. Wenn man abends um 19 Uhr müde nach Hause kommt, dann verschiebt man das Forschen aufs Wochenende, da braucht man aber eher Erholung. In der Weiterbildungsphase die Doktorarbeit zu schreiben ist also nicht sehr günstig, auch wenn es auch hier hin und wieder den für mich erstaunlichen Fall gibt, dass es jemand schafft. Aber es ist eher selten, dass das gelingt.

\section{Wie findiet man das richtige Thema für seine Doktorarbeit?}

Ich hatte eine angehende Doktorandin zu Besuch, die habe ich das auch gefragt. Sie sagte mir, unter ihren Kommilitonen gelte es als ganz besonders wichtig, wer Doktorvater oder Doktormutter wird, der oder die sich dann auch um das Thema und seine Betreuung kümmert. Die Persönlichkeit des Betreuers spielt bei der Themenwahl also offenkundig eine überragende Rolle. Dann geht es natürlich auch noch um die Frage: Für was für eine Art von Doktorarbeit eigne ich mich? Es beginnt mit retrospektiven klinischen Studien, bei denen schon vorhandenes Datenmaterial mit fraglicher Qualität der Daten analysiert wird. Diese Arbeiten gelingen in der Regel, denn sie sind relativ leicht zu bewältigen, allerdings auch wenig spannend, da man hier ja keine Hypothese beweisen, sondern allenfalls eine Hypothese generieren kann.

Anders ist es mit klinisch-prospektiven oder mit epidemiologischen Arbeiten, bei denen man die Daten erst gewinnen muss. Das kann länger dauern als man zunächst glaubt. Ähnlich ist es bei einer experimentellen Arbeit. Nur bei einer prospektiven und experi- 
mentellen Arbeit ist es möglich, eine These zu bestätigen oder zu entkräften. Das gilt als wissenschaftlich wertvoller, wird dann auch mit besseren Noten wie «magna cum laude» oder «summa cum laude» belohnt. Solche Arbeiten haben aber auch das Risiko, dass sie scheitern, weil nicht genügend Patienten zur Untersuchung vorhanden sind, der Beobachtungszeitraum zu kurz ist oder sich die Hypothese nicht bestätigen lässt.

Schließlich gibt es auch noch «Literaturarbeiten», bei denen man auch eine sehr gute Note bekommen kann. Allerdings sind diese Arbeiten - besonders in meinem Fachgebiet Geschichte, Theorie und Ethik der Medizin - methodisch vom naturwissenschaftlich dominierten Medizinstudium recht weit entfernt, sodass es dann auch nicht schadet, wenn jemand sprachliche, philosophische, juristische oder theologische Interessen beziehungsweise Vorkenntnisse hat.

Man sollte sich also zunächst einmal überlegen, wo die eigene Neigung hingeht, und dann auch eruieren, was an der Medizinischen Fakultät angeboten wird. Viele Betreuer haben eine aussagekräftige Homepage, auf der man erkennen kann, was da früher schon an Doktorarbeiten betreut worden ist, wie die Themen lauten und was die Forschungsschwerpunkte sind. Man sollte sich auf jeden Fall kundig machen, bevor man den direkten Kontakt sucht, weil Doktorväter und -mütter es natürlich gerne sehen, wenn ein potenzieller Doktorand eine gewisse Vorstellung hat, was in dem entsprechenden Bereich, in der Abteilung oder in der Klinik geforscht wird.

Ist es notwendig, die Promotion in dem Bereich zu machen, in dem später auch die Weiterbildung absolviert wird?

Nein, sicher nicht. Ich selbst, der ich heute Medizinhistoriker, Wissenschaftsphilosoph und Medizinethiker bin, habe 1979 in der Hals-Nasen-Ohren-Klinik in Freiburg über die stereophone Hörgeräteversorgung promoviert. Oder denken Sie an die Fächer des Grundstudiums, also Anatomie, Physiologie und Biochemie. Da promovieren nicht nur Kollegen, die später Physiologe oder Biochemiker werden wollen. Es ist durchaus möglich, in dem Fach, auf das man sich später spezialisiert, bereits die Dissertation zu schreiben. Aber die Promotion ist keine Vorentscheidung in dem Sinn, dass man danach nichts anderes mehr machen könnte.

\section{Kommen wir zu den praktischen Tipps: Ist es sinnvoll die Literatur} mit einem Literaturverwaltungsprogramm zu katalogisieren?

Wenn man beginnt, Literatur zu sammeln, ist es wichtig, erst einmal zu schauen, wie die entsprechenden Zitierregeln an der jeweiligen Fakultät sind. Das heißt, bestimmte Zitierweisen werden an einer Medizinischen Fakultät vorgeschrieben, während an einer anderen Fakultät wieder ganz andere Regeln verpflichtend sind. An diese Regeln sollte man sich von Anfang an halten, gleichgültig, ob man ein Literaturverwaltungsprogramm verwendet wie EndNote oder Citavi oder wie sie alle heißen mögen.

Man kann auch ohne ein Literaturverwaltungsprogramm arbeiten. Ich selbst z.B. mache meine Literaturverwaltung grundsätzlich von Hand, und das, obwohl gerade ein Medizinhistoriker und Medizinethiker sehr viel Literatur kennen und zitieren muss. Unabhängig davon, wie man die gelesene Literatur verwaltet, sollte man das Gelesene von Anfang an gut dokumentieren, weil man ja doch unterschätzt, was man alles wieder vergisst. Wenn man sich von Anfang an Notizen gemacht und die Literatur gesammelt hat, dann hat man es später leichter.

\section{Gibt es an den Universitäten Hilfestellung jenseits der betreuenden Professoren?}

Ja, die gibt es, z.B. bei uns an der Medizinischen Fakultät Mannheim der Universität Heidelberg. Wir haben seit dem Wintersemester 2015/16 im 3. Studienjahr den obligatorischen Leistungsnachweis «Wissenschaftliches Arbeiten». Diesen Leistungsnachweis müssen alle Studierenden erbringen. Zum Abschluss müssen die Studierenden - spätestens bis zum Ende des 5. Studienjahres - eine Forschungsarbeit von etwa 15 Seiten schreiben. Das ist eine Vorstufe zur Doktorarbeit, da kann man sich schon den Betreuer aussuchen, bei dem man später vielleicht promovieren will und fertigt unter seiner Leitung eine Forschungsarbeit an. Diese Arbeit wird vom Betreuer in 7 Dimensionen benotet, und aus den besten Absolventen wählt man als künftiger Doktorvater seine Doktoranden leichter aus als früher. Das hat sich bei uns in den letzten 2 Jahren schon sehr bewährt, denn dadurch bekommt die Wissenschaftlichkeit schon im Hauptstudium einen hohen Wert.

Die Promotion ist keine Pflicht. Wann würden Sie einem Studierenden, der zu Ihnen mit einem Promotionswunsch kommt, von dem Schreiben einer Dissertation abraten?

Da haben wir in Mannheim fast schon ideale Verhältnisse, dass nämlich alle Studierenden eine Forschungsarbeit anfertigen müssen. Wenn jemand bei mir bei der Forschungsarbeit schon sehr gut ist, kann er danach eine Doktorarbeit übernehmen. Wenn jemand bei der Forschungsarbeit aber zu der Erkenntnis kommt, dass es ihm keinen Spaß gemacht hat oder dass sie ihm nicht gut gelungen ist, dass eher die Notwendigkeit als der Wunsch nach einer wissenschaftlichen Arbeit zielführend war, dann würde ich eher abraten. Denn nichts wäre frustrierender, als wenn man sich 2, 3 oder gar 4 Jahre durch eine Doktorarbeit quält, nur um den Doktorgrad zu bekommen.

Man kann nie im Voraus sicher sagen, wenn jemand eine Promotion beginnt, was dabei herauskommt. Aber ich würde sagen, etwa 20\% der Studierenden überschätzen sich und brechen ihr Vorha- 
ben schließlich ab. Man darf nicht vergessen, sie sind meistens Anfang bis Mitte zwanzig. Da gibt es biographische Brüche, oder es wird einem eine Stelle als Assistenzärztin oder Assistenzarzt angeboten, und dann hat sie oder er keine Zeit mehr für eine Dissertation. Das alles muss man einkalkulieren.

Was ist die wichtigste Charaktereigenschaft, die man benötigt, um eine Doktorarbeit erfolgreich abzuschließen?

Selbstdisziplin ist im Berufsleben insgesamt sehr viel wichtiger, als man vielleicht in jungen Jahren meint. Dass man sich eben jeden Tag oder zu bestimmten Zeiten hinsetzt und sagt: So, jetzt schreibe ich an meiner Doktorarbeit.

Nehmen wir an, es gibt einen sehr disziplinierten Studenten, der das klare Ziel hat, die Promotion abzuschließen. Wie viel Zeit muss er dem Vorhaben einräumen?

Zunächst muss man das reguläre Studium mit dem Ziel des Staatsexamens bewältigen, sodass sich die Arbeit an der Dissertation auf die Wochenenden und die Semesterferien konzentrieren wird. Man kann sich die Daten der Semesterferien aufschreiben und einen Plan machen, doch dann kommen Prüfungen dazwischen und vieles andere. Aber es empfiehlt sich in jedem Fall, eine bestimmte Zeit für die Doktorarbeit zu reservieren. Ob das nun jeden Tag ist, das wird man gar nicht durchhalten, denn man muss realistisch bleiben. Es gibt auch hier Zeiten, zu denen man nicht arbeiten will oder kann. Aber man kann sich vorher überlegen, wie viel Zeit bis zum Staatsexamen noch bleibt. Reicht das, um die Dissertation im Manuskript abzuschließen, oder ist das von Vornherein unrealistisch?

Was spricht Ihrer Meinung nach für einen Doktorgrad - gesellschaftliches Ansehen, besserer Verdienst?

Letzteres sicher nicht. Ist man im Krankenhaus angestellt, so bekommt man das gleiche Gehalt, unabhängig davon, ob man pro- moviert ist oder nicht. Allerdings, wenn man später beabsichtigt, Oberarzt oder Chefarzt zu werden, dann kommt man um eine qualifizierte Promotion nicht herum, weil die Karriere sonst rasch zu Ende geht. Denn man wird immer promovierte - und inzwischen auch qualitativ hochwertig promovierte - Kolleginnen und Kollegen haben, die dann den Vorzug bekommen. Das hohe gesellschaftliche Ansehen des Arztes ist traditionell aber schon noch ein wichtiges Motiv, denn mit dem Doktorgrad assoziiert man immer noch vor allem den Mediziner. Erst allmählich beginnt sich dieses Stereotyp zu wandeln, und das ist gut so.

\section{Zum Autor}

Prof. Dr. med. Axel W. Bauer leitet heute das Fachgebiet Geschichte, Theorie und Ethik der Medizin an der Medizinischen Fakultät Mannheim der Universität Heidelberg. Seine Promotion machte er dagegen in der Hals-Nasen-Ohren-Klinik in Freiburg. Mit dem Ratgeber «Promotion: Die medizinische Doktorarbeit - von der Themensuche bis zur Dissertation» (ed 4., Stuttgart, Thieme, 2015) wollen die Autoren Christel Weiß und Axel Bauer Studierenden der Medizin das Schreiben der Doktorarbeit erleichtern.

Kontaktadresse: Prof. Dr. med. Axel W. Bauer, Fachgebiet Geschichte, Theorie und Ethik der Medizin, Medizinische Fakultät Mannheim der Universität Heidelberg, Ludolf-Krehl-Straße 13-17, Haus C, Ebene 6, Raum 614, 68167 Mannheim, Deutschland, axel.bauer@medma.uni-heidelberg.de.

\section{Originalquelle}

Experte im Gespräch: Prof. Axel W. Bauer über die medizinische Promotion; in Operation Karriere - Das Karriereportal und der Kongress für angehende und junge Ärzte. www.operation-karriere.de. (Direktlink für Onlineversion: http://www. operation-karriere.de/karriereweg/medizinstudium/experte-im-gespraech-profaxel-w-bauer-ueber-die-medizinische-promotion.html)

\section{Junge Ärzte \\ Praxis-Alltag: Das Problem mit der Zeit kennt jeder Arzt}

Bei einem Netzwerktreffen in München haben junge Ärzte sich ausgetauscht und aktuelle Probleme besprochen. Sie alle wünschen sich mehr Zeit, um ärztlich zu arbeiten.

München. Mehr Zeit, um ärztlich zu arbeiten - dieser Wunsch stand beim Treffen des Bündnisses Junge Ärzte (BJÄ) im Vordergrund. Beraterfirmen, die minutengenaue Vorgaben für Patientenvisiten machen, Geschäftsführer, die ärztliche Maßnahmen nur als abzurechnende Einheiten sehen: Solche Erfahrungen haben viele der jungen Fachärzte in Kliniken schon gemacht. Das sei schlecht für Patienten und Ärzte, so ihre Kritik.

«Ärztliches Arbeiten ist nicht, in zwei Minuten die Liste abzuhaken. Es ist sehr viel mehr», betonte Dr. Kevin Schulte, Sprecher des BJÄ und Vertreter der außerordentlichen Mitglieder im Vorstand des Berufsverbandes Deutscher Internisten (BDI). Erste Aufgabe von Ärzten sei es, zu «verstehen, wer vor einem sitzt». Das sei aber nur im persönlichen Gespräch mit Patienten möglich. Dieser Austausch sei für den Behandlungserfolg letztlich ebenso wichtig wie die fachliche Seite.

\section{Arzt als Berater des Patienten}

Für eine aufgeklärte Entscheidung müssten Patienten zuerst die Optionen verstehen. Ob es nun um einen Herzkatheter gehe, oder um die Form der Narkose: Patienten wünschten sich, dass ihre Fragen beantwortet werden. Es gehöre zum Arztsein, ihnen ihre Unsicherheit zu nehmen. «lch verstehe mich als Ärztin auch als Bezugsperson und Berater des Patienten», so Dr. Anne Blank. Die Anästhesistin vertrat die jungen Ärzte in der Deutschen Gesellschaft für Anästhesiologie und Intensivmedizin (DGAI).

Leitlinien erfüllten ihren Sinn nur, wenn der Arzt ihre Inhalte individuell auf die Situation anwendet. Werde statt dessen ein mechanistisches Abfertigen von Patienten erzwungen, führe das jede Leitli- 
nie ad absurdum. «Das führt dazu, dass das Problem woanders im Gesundheitswesen wieder aufploppt», gab Mira Mikhail von der German Society of Residents in Urology (GeSRU) zu bedenken.

Eine kurzsichtige, rein ökonomisch orientierte Strategie erweise sich am Ende auch für das GKV-System als Nachteil. Es untergrabe die ärztliche Autonomie, wenn nur Ökonomen die Klinik-Prozesse steuerten. «Ärzte müssen an wichtigen Entscheidungsprozessen weiter beteiligt werden», forderte Mikhail. Im besten Fall sollten Klinikleiter sowohl ärztliche als auch wirtschaftliche Kompetenz haben, konstatierte Schulte. «Wenn ein Arzt beides macht, wird er zurückhaltender sein, Zwölf-Prozent-Rendite-Ziele zu formulieren», ist sich der Internist sicher.

Dass Innovationen mehr Zeit im Arbeitsalltag verschaffen können, berichtete Dr. Jürgen Konczalla von der Deutschen Gesellschaft für Neurochirurgie (DGNC). An der Klinik für Neurochirurgie der Universitätsklinik Frankfurt übernähmen inzwischen Physicians Assistants viele Aufgaben in der Dokumentation. Eine elektronische Patientenakte ermögliche es zudem, Laborwerte oder Radiologiebilder jederzeit direkt auf den eigenen Bildschirm zu holen. «Das hat uns sicher geholfen, knappe Ressourcen sinnvoll zu nutzen», so der Neurochirurg. Nicht zuletzt hätten Ärzte dadurch mehr Zeit für ihre Patienten. Ob solche Lösungsansätze die ärztliche Arbeit am Ende verbesserten, hänge aber von der Klarheit der ärztlichen Rolle ab, stellte Schulte fest. Als erstes müssten sich Ärzte darüber klar sein, welche Aspekte originär zu ihrer Tätigkeit gehörten. Diese dann durch digitale Mittel, Kooperation oder Delegation effizienter zu gestalten, sei der zweite Schritt. Die Ärzteschaft müsse sich intensiver mit dem Bild vom eigenen Arztsein auseinandersetzen. Mehr Engagement wünschten sich die jungen Fachärzte dazu unter anderem von den Ärztekammern.

\section{Geschäftsführer für alle Fälle}

Die Niederlassung als Vertragsarzt könne ein Weg sein, die Arbeit eigenständiger zu gestalten. Für sich selbst nannte immerhin jeder zweite der 19 Teilnehmer diesen Schritt als relevante Option. Mit eigener Praxis hätten Ärzte fachliche und wirtschaftliche Zuständigkeit - das kann Vorteil und Nachteil zugleich sein. Denn auf die ökonomische Seite der Praxisführung seien junge Ärzte oft nicht gut vorbereitet. Das gelte auch für die Auswahl und das Führen von Mitarbeitern. Auch das Regressrisiko und ein mögliches Scheitern der Praxis wird von vielen als bedrohlich wahrgenommen.

Insgesamt, so die Meinung einiger Teilnehmer, sei vieles am kassenärztlichen System undurchsichtig. «lch würde mir wünschen, dass die KVen dieses komplexe System einfacher und transparenter darstellen», so Schulte. Hier erwarteten die jungen Ärzte mehr Impulse von den Berufsverbänden. Das könne dazu beitragen, mehr Mediziner zur Niederlassung zu motivieren. Am vielversprechendsten erschienen den Teilnehmern dabei gemeinschaftlichen Praxisformen - gerne auch mit angestelltem Geschäftsführer für die wirtschaftlichen Fragen. Dr. Benedikt Braun, Vertreter des Perspektivforums junge Chirurgie der Deutschen Gesellschaft für Chirurgie (DGCH), konstatierte: «Die Verantwortung ist auch bei den Ärzten selbst, wie sie ihre Tätigkeit gestalten, und sich dafür den Raum schaffen.»

Christina Bauer, Journalistin u. Autorin, München, Deutschland

\section{Originalquelle}

Junge Ärzte: Das Problem mit der Zeit kennt jeder Arzt; in: «Ärzte Zeitung», Nr. 227 vom 27. November 2017, Seite 6, Springer Medizin Verlag GmbH (Direktlink für Onlineversion: www.aerztezeitung.de/948198) 


\section{Wissenschaftliches Publizieren}

\section{(Warum) brauchen wir Verlage?}

Die Aufgaben eines Verlags werden immer sein: Das Sammeln, Sichten, Auswählen, Verdichten, Verbreiten und Bewahren von Informationen in allen Formen und auf jede erdenkliche Weise. Dies sagte schon mein Urgroßvater vor 127 Jahren bei der Gründung des Karger Verlags. Und das gilt noch heute: Wissenschaftliche Verlage haben mehr denn je die Aufgabe des Vermittelns und Vernetzens zwischen den verschiedenen Akteuren der Wissenschaft. Sie selektieren Inhalte und bieten damit Orientierung in einer immer größer werdenden Flut an Informationen. Die Verlage organisieren das Peer Reviewing, das eine wissenschaftlich hochstehende Qualität gewährleistet. Außerdem brin-

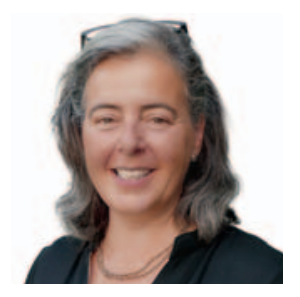

Gabriella Karger Delegierte des Verwaltungsrats \& Verlegerin, S. Karger AG gen Verlage den Inhalt durch Lektorat und Layout in eine stringente und ansprechende Form. Ein wichtiger Aspekt ist auch das Be- wahren der Informationen, das Archivieren, gerade in der digitalisierten Wissensgesellschaft. Ein weitgehend «unsichtbarer» Service von Verlagen ist das Gewährleisten der Auffindbarkeit von wissenschaftlichen Artikeln durch das aktive Verteilen von Metadaten an relevante Suchmaschinen sowie Abstracting und Indexing-Datenbanken. Dank neuer technischer Möglichkeiten gewährleisten sie auch semantische Verknüpfungen, die überraschende Entdeckungen ermöglichen. Dadurch wird sichergestellt, dass die Artikel von anderen Wissenschaftlern gefunden werden und wiederum in deren Forschung einfließen können. Verlage leisten damit einen Beitrag zum wissenschaftlichen Diskurs und Erkenntnisfortschritt und sind dadurch sowohl Dienstleister als auch Partner der Wissenschaft.

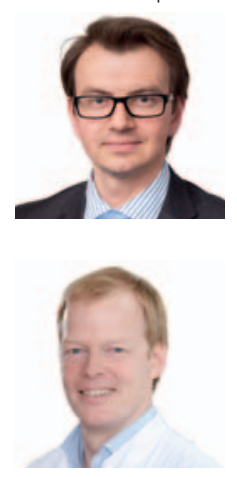

_ Prof. Dr. Matus Rehak

Herausgeber Karger Kompass Ophthalmologie

Ja, denn so kann jeder Wissenschaftler seine Untersuchungen einreichen und sicher sein, dass diese durch ein unabhängiges Begutachtungsverfahren (Peer-review) geprüft werden. Außerdem muss ich mich als Autor nicht mit den verlegerischen Aspekten, wie Lektorat, Gestaltung etc. beschäftigten, sondern ich weiß, dass der Verlag sich kümmert.

\section{—Prof. Dr. Nicolas Feltgen}

Fachbeirat Karger Kompass OphthaLmologie

Ja, wir brauchen Verlage, denn sie sind in der Lage, Informationen entsprechend den Interessen der Leser und Autoren zu bündeln. Die Qualität dieser Beiträge wird zudem durch ein Peer-Review geleistet, das von den Verlagen organisiert und koordiniert wird. Dadurch wird nicht zuletzt die wissenschaftliche Qualität auf einen Nenner und vergleichbar gemacht. Insbesondere bei KARger Kompass wird die Qualität der Arbeit durch die kritische Auseinandersetzung im Wissenstransfer auf einer praxisorientierten Ebene geprüft. So werden auch über Verlage Diskussionen angeregt, die über den rein wissenschaftlichen Kontext hinausgreifen.

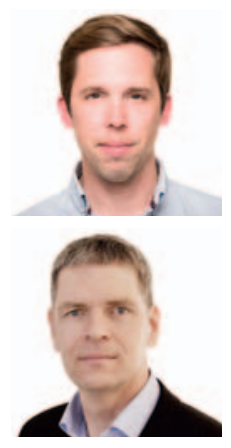

- Dr. Matthias Raspe

Koordination «PneumoCampus», Karger Kompass PNeumologie

Bei der unüberschaubaren Menge und Fluktuation von Fachinformationen, wie wir sie heute vorfinden, sind Verlage von unschätzbaren Wert. Sie ordnen und bereiten Fachwissen auf und machen es somit erst wirklich nutzbar.

\section{- Bernd Adam}

Geschäftsführer Deutsche Fachpresse

Die heutige Wissenschafts- und Arbeitswelt ist dadurch gekennzeichnet, dass Informationen im Überfluss vorhanden sind und die Menge rasant wächst. Welche Informationen aber sind relevant für den einzelnen Menschen? Wie sind die Informationen zu bewerten? Und worauf ist wirklich Verlass? Verlage bieten mit ihren Produkten fundierte Einordnung durch Expertise, wissenschaftliche Standards und hohe journalistische Qualität. Sie machen aus Informationen relevantes Wissen und geben Orientierung. Dabei sind sie nicht nur als verlässliche Informationsquellen, sondern auch als unabhängige Meinungsträger essenziell. Kurz: Ja, wir brauchen Verlage, denn ihre Arbeit ist unverzichtbarer Bestandteil einer wissensstarken und freien Gesellschaft.

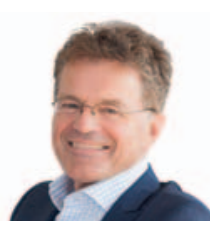

\section{-Alexander Skipis}

Hauptgeschäftsführer des Börsenvereins des Deutschen Buchhandels

Verlage sind Garanten für die Qualität und Vielfalt des deutschen Buchmarktes und tragen wesentlich zum Gelingen einer freien, demokratischen Gesellschaft bei. Verlage unterstützen Autorinnen und Autoren im gesamten Veröffentlichungsprozess, sie geben ihren Ideen eine Form und bringen sie gestützt durch Lektorat, Herstellung, Marketing und Vertrieb an den Leser. Mit ihrer Arbeit tragen Verlage zur Verbreitung von Ideen und zur Meinungsvielfalt bei. Sie stoßen Debatten an und leisten damit einen wichtigen Beitrag zum kulturellen und gesellschaftlichen Leben. 